\title{
PyramidApp: Scalable Method Enabling Collaboration in the Classroom
}

\author{
Kalpani Manathunga, Davinia Hernández-Leo \\ ICT Department, Universitat Pompeu Fabra, Barcelona, Spain \\ \{kalpani.manathunga, davinia.hernandez\}@upf.edu
}

\begin{abstract}
Computer Supported Collaborative Learning methods support fruitful social interactions using technological mediation and orchestration. However, studies indicate that most existing CSCL methods have not been applied to large classes, means that they may not scale well or that it's unclear to what extent or with which technological mechanisms scalability could be feasible. This paper introduces and evaluates PyramidApp, implementing a scalable pedagogical method refining Pyramid (aka Snowball) collaborative learning flow pattern. Refinements include rating and discussing to reach upon global consensus. Three different face-to-face classroom situations were used to evaluate different tasks of pyramid interactions. Experiments led to conclude that pyramids can be meaningful with around 20 participants per pyramid of 3-4 levels, with several pyramids running in parallel depending on the classroom size. An underpinning algorithm enabling elastic creation of multiple pyramids, using control timers and triggering flow awareness facilitated scalability, dynamism and overall user satisfaction in the experience.
\end{abstract}

Keywords: Computer-Supported Collaborative Learning, Pyramid / Snowball Collaborative Learning Flow Pattern, Large Groups, Classroom

\section{Introduction}

Multiple findings from educational research highlight the importance of active learning [1]. In particular, sound collaborative learning methods foster rich social interactions between students leading to fruitful learning. Provision of technological means to support collaboration has enabled new or enhanced learning scenarios [2]. Technologies can mediate social interactions; facilitate orchestration regarding coordination requirements (e.g., group distribution); monitor interactions for regulation. Yet, despite the potential technologies, effective CSCL methods that favour equal, meaningful interactions between students -sometimes referred as macro-scripts [3,4]-, have been mostly applied upon small groups of students [5].

Recently, popularity and social impact of open educational settings such as Massive Open Online Courses (MOOCs) have driven more research interests around scalable pedagogies [6] and urge to build up pedagogical methods based on active learning approaches fostering social interactions [7]. Unstructured discussion through forums and social media helps [7], but its potential effectiveness is limited compared to

adfa, p. 1, 2011.

(C) Springer-Verlag Berlin Heidelberg 2011 
what can be achieved by more structured CSCL approaches [3]. The need for active learning in large classroom settings has been acknowledged for over three decades [8]. However, actual teaching practice in large classrooms is still broadly based on lecturing with passive participation of students. Only few remarkable initiatives have offered technological solutions to facilitate active learning in large classroom, based on collective polls or self-organized backstage interactions $[9,10]$. However, there are no approaches that extrapolate sound macro-scripts methods that structure the collaborative learning flow for effectiveness in terms of fostering individual accountability, positive interdependence and meaningful interactions between students [3, 4].

Direct application of collaborative learning methods that work well with small groups appears to be challenging in both massive virtual learning contexts and large synchronous classes due to lack of scalable aspects or practical challenges hindering sensible implementation of CSCL methods. Practical challenges include time and teachers' load limitations if learning outcomes of all groups should be measured in a large classroom or implications of flexibility issues with large and varying number of students [2]. This research work aims at exploring to what extent or with which technological mechanisms, scalability of relevant collaborative learning methods could be feasible. In particular, the paper studies the Pyramid (a.k.a. Snowball) method, which intuitively suggest reasonable scalability potential. Pyramid method, formulated as a Collaborative Learning Flow Pattern (Pyramid CLFP), can be particularized and reused with multiple epistemic tasks and educational levels [4]. A Pyramid flow starts with individual proposals being discussed and iteratively joined into larger-groups till a common consensus is reached upon at the global level. Such scenarios foster individual participation and accountability (equal opportunity for all, yet with singular contributions) and balanced positive interactions (opinions of all members count) in a collaborative knowledge-oriented negotiation process. The specific research question that guides this research is how can the Pyramid flow pattern be technologically supported to serve as a scalable method for collaboration in the classroom?

To tackle the question, we have iteratively proposed, prototyped and evaluated refinements of the Pyramid CLFP. Initial refinements propose a method using peer rating promoting integrated consensus reaching accompanied by discussion. The technological implementation of this is "PyramidApp" tool. Main challenges identified at initial iterations referred to scalability and dynamism. With scalability we mean capability to elastically accommodate growing numbers of participants while maintaining pedagogical and practical effectiveness. As dynamism we mean the ability to keep activity progression while preserving enthusiasm and usability. To achieve scalability and dynamism, iterative refinements of the method incorporate an algorithm implementing flow creation, flow control and flow awareness rules. A first evaluation of PyramidApp in three real-class contexts offers insights about its scalability prospects and suitability of the proposed rules. 


\section{PyramidApp method and algorithmic rules}

PyramidApp is a technological solution implementing a scalable method applying Pyramid CLFP principles. Individuals propose their option (i.e. can be a question or an answer for a given task, seeking active comprehension) and PyramidApp forms small groups to share ideas about suggested options, to clarify and negotiate before rating the options. Highly rated options are promoted to upper levels and groups grow larger (by joining previous smaller groups) following a pyramid/snowball structure. Rating and discussion propagate till the final level until the complete group reaches upon a global consensus on the best options. Everyone, including the educator, see finally selected options to which the educator comments. In large classes, educators do not have sufficient time to comment each individual's option (questions or answers), but can attend for an agreed selection of options more feasibly. At the same time, all students have the chance to express and discuss their ideas and to critically reflect and assess peer's contributions, with positive benefits in their negotiation skills and knowledge building process. Initial prototypical pyramid implementations were developed and evaluated in rounds to iterate behavioural rules for the algorithm behind the method to address scalability and dynamism issues (Fig.1). Flow creation rules allow scalability by automatically adapting the pyramid structure based on the number of joining participants providing an elastic mechanism of multiple pyramid creation. Flow control rules lead to dynamism by preventing potential blocking within flow progression if participants leave (due to any reason: unexpected situation or technological problem). Parameter values presented in Fig.1 are shown with recommended values that are configurable if preferred. Those estimations were acquired from the initial evaluations. For example, it was observed that when number of level increases participants absolve enthusiasm, longer timing values lead to boredom, very high satisfaction percentages may froze pyramid branches or lead to higher waiting time. Flow awareness rules (e.g.: progression level, group members, timing notifications and selected options along the flow) elevate learner engagement and usability.

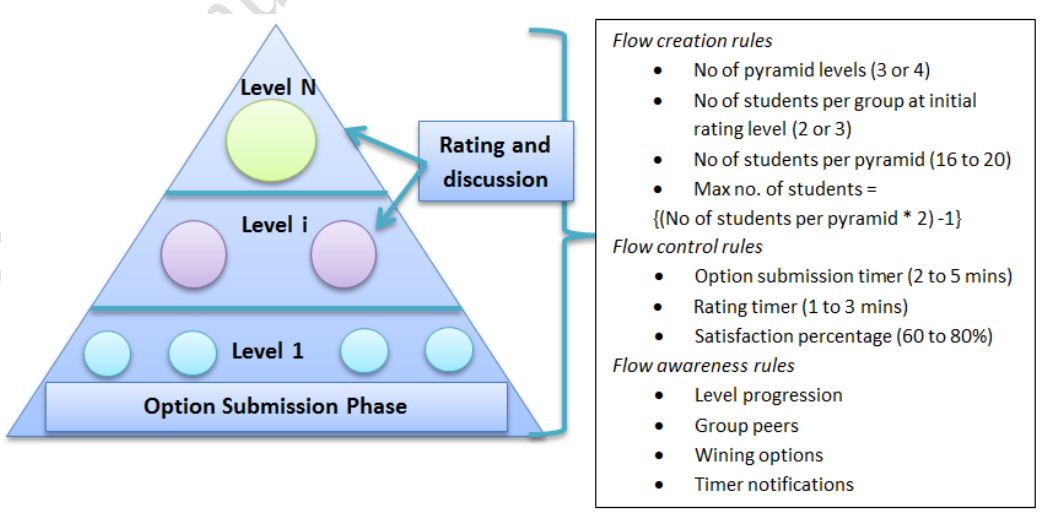

Fig. 1. Schema of PyramidApp: parameters for flow creation, control and awareness rules 


\section{Evaluation}

PyramidApp's algorithmic rules were evaluated for potential scalability (ability to accommodate an increase in the number of participants) and dynamism (ability to keep activity progression) while maintaining pedagogical and practical effectiveness alongside enthusiasm and usability. The evaluation also seeks which configurations of the method (values for parameters in rules and to use discussion or not) achieve satisfactory scalability, dynamism and overall impact. Across all experimental settings, $80 \%$ satisfaction percentage was maintained and deliberately enabled or disabled chat to observe different behaviour. Table 1 explains the nature of each experimental setting, tasks given and pyramid configuration parameters with values. Graphs illustrate timing aspects across three settings.

Table 1. Pyramid configurations and timing aspects across three experimental settings

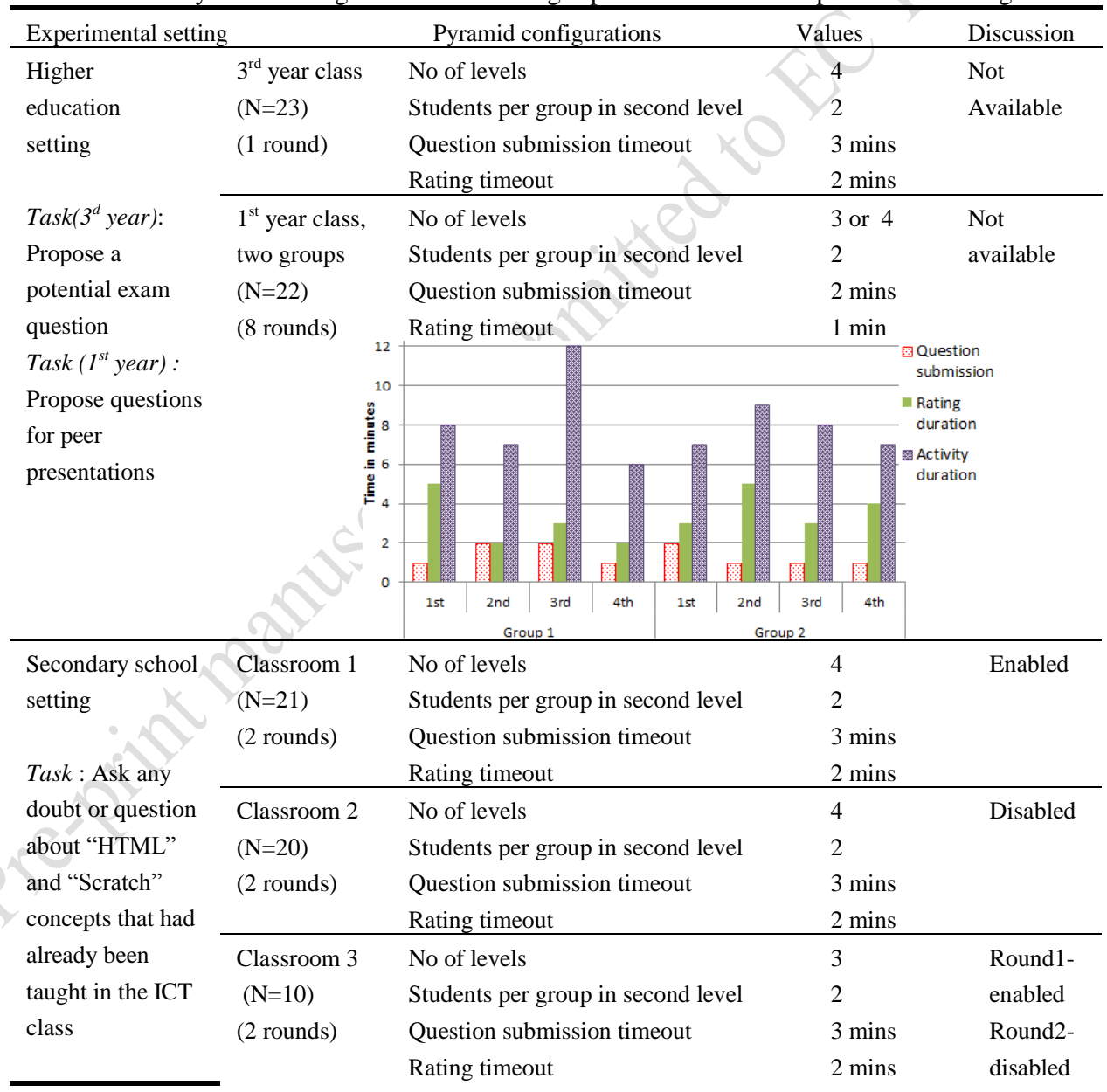




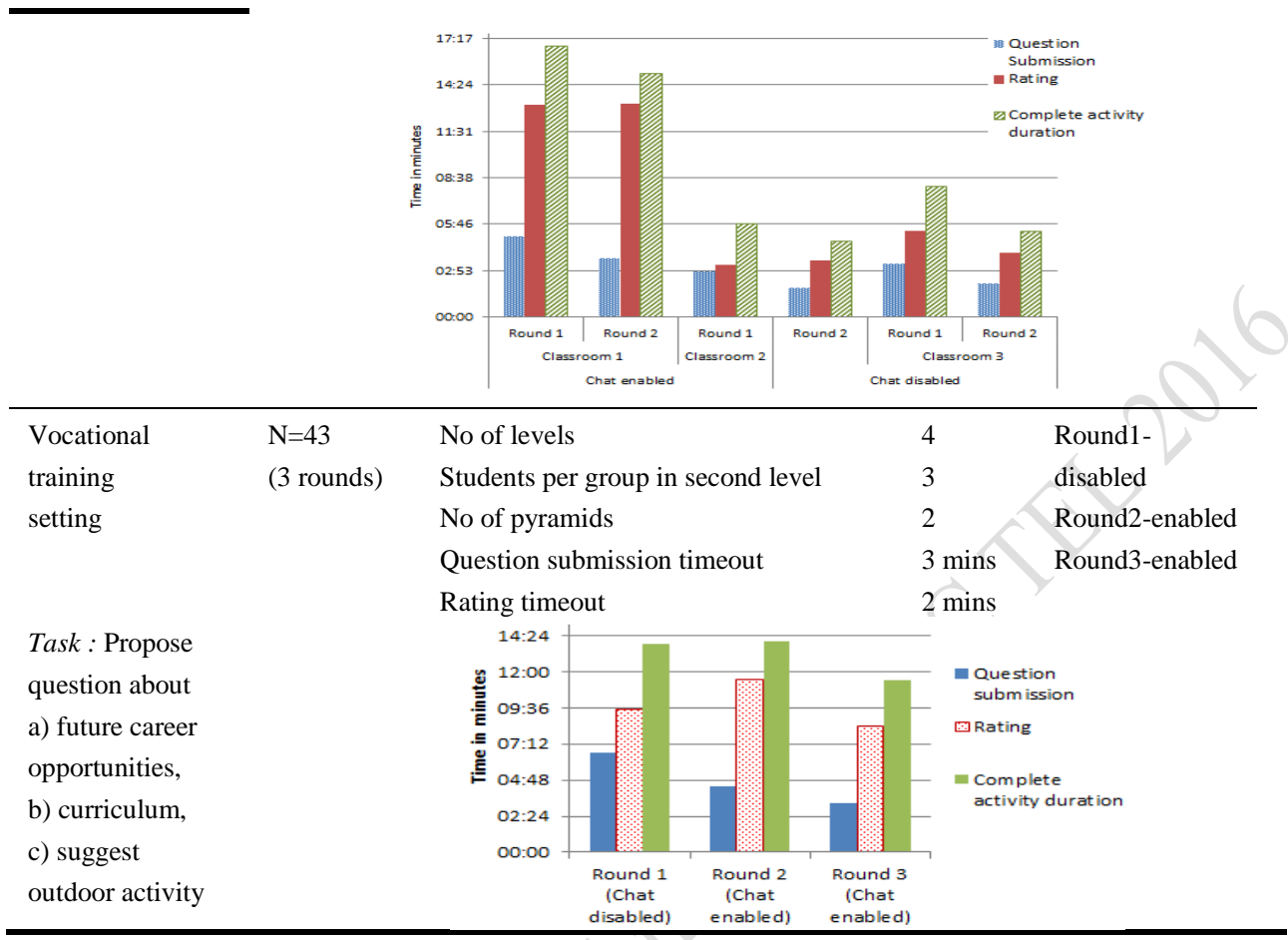

Proposed Pyramid refinement satisfactorily accommodates groups of up to 20 students in a single pyramid and several pyramids can run in parallel in large classrooms (two pyramids in the vocational training setting) facilitating late comers to join ongoing activities or managing drop-outs. Each Pyramid flow results a single outcome that can be commented by the educator. A classroom of 100 students will have five outcomes, which can be feasibly addressed. Pyramids of 3 or 4 levels can maintain satisfactory engagement. Flow control rules like timers facilitated dynamism by preserving activity progression. Depending on the context, a pyramid activity can take between 5 to 16 minutes. Table 2 shows structural scalability and adapted dynamism.

Table 2. Dynamism and scalability preservation in Pyramid flows within three settings

\begin{tabular}{|c|c|c|}
\hline Experiment Setting & Structural aspects & Comments / Observations \\
\hline $\begin{array}{l}\text { Higher } \\
\text { education } \\
\text { setting }\end{array}$ & $\begin{array}{l}\text { Timeouts : } 5 \\
\text { Late logins : } 1\end{array}$ & $\begin{array}{l}\text { Pyramid flows were not frozen or interrupted thanks to } \\
\text { flow control mechanisms (satisfaction percentage and } \\
\text { timers) maintaining dynamism and flow creation rules } \\
\text { enabling a scalable inclusion of students joining late. }\end{array}$ \\
\hline $\begin{array}{l}\text { Secondary school } \\
\text { Setting }\end{array}$ & Late logins: 7 & $\begin{array}{l}\text { Flow control rules (timers and satisfaction percentage) } \\
\text { allowed smooth flows irrespective of multiple timer expi- } \\
\text { rations maintaining dynamism. Students were enthusiasti- } \\
\text { cally and participating in discussions and rating. }\end{array}$ \\
\hline $\begin{array}{l}\text { Vocational training } \\
\text { Setting }\end{array}$ & $\begin{array}{l}\text { Desired pyramid sizes } \\
\text { Final pyramid sizes }\end{array}$ & $\begin{array}{llll}\text { Round 1 } & \text { Round 2 } & \text { Round 3 } & 2 \text { pyramids were } \\
20 \& 16 & 20 \& 16 & 20 \& 16 & \text { enacted without } \\
20 \& 19 & 20 \& 27 & 20 \& 22 & \text { interruptions. }\end{array}$ \\
\hline
\end{tabular}


As with any pedagogical method, its effectiveness depended on the context (e.g., classroom atmosphere) and proposed epistemic tasks (e.g., need of active comprehension). Along with dynamism, flow awareness rules contributed to preserve enthusiasm and usability. Rating, viewing winning options and levelling up in pyramid offering gaming effect were perceived with more than $85 \%$ satisfaction across all experiments.

\section{Conclusion}

Diverse educational contexts raise requirements for active pedagogical methods like collaborative learning to be applied with large numbers of students using reasonable time durations. In the paper we identified scalability and dynamism as the key requirements to be addressed by such methods and their technological implementations. We studied a refined implementation (PyramidApp) of the Pyramid flow, addressing these issues, incorporating flow creation, flow control and flow awareness rules. Results suggest suitability of the mechanisms behind the method and open new perspectives that are worth further exploring with diverse epistemic tasks, contexts, larger classroom settings and other challenging settings such as massive open courses.

Acknowledgements. Special thanks to participants from Escola Forestal De Sta. Coloma De Farners, Oak House School and Engineering School of UPF Barcelona. This work has been partially funded by the Spanish Ministry of Economy and Competitiveness (TIN2014-53199-C3-3-R; MDM-2015-0502).

\section{References}

1. Bonwell, C. C., \& Eison, J. A.: Active Learning: Creating Excitement in the Classroom. ERIC Clearinghouse on Higher Education, The George Washington University, Washington, DC (1991)

2. Dillenbourg, P., \& Tchounikine, P.: Flexibility in macro-scripts for CSCL. Journal of Computer Assisted Learning. 23(1):1-13(2007)

3. Dillenbourg, P.: Split where interaction should happen, a model for designing CSCL scripts. In P. Gerjets, PA Kirschner, J. Elen \& R. Joiner (Eds.), Instructional design for effective and enjoyable computer-supported learning. Tuebingen: Knowledge Media Research Centre (2004)

4. Hernández-Leo, D., Villasclaras-Fernández, E. D., Asensio-Pérez, J. I., Dimitriadis, Y., JorrínAbellán, I. M., Ruiz-Requies, I., \& Rubia-Avi, B.: COLLAGE: A collaborative Learning Design editor based on patterns. Journal of Educational Technology and Society, 9(1), 58-71(2006)

5. Manathunga, K., \& Hernández-Leo, D.: Has research on collaborative learning technologies addressed massiveness? Journal of Educational Technology \& Society. 18 (4), 357-370 (2015)

6. Ferguson, R., \& Sharples, M.: Innovative pedagogy at massive scale: teaching and learning in MOOCs. In: Open Learning and Teaching in Educational Communities, pp. 98-111. Springer (2014)

7. Rosé, C.P., Carlson, R., Yang, D., Wen, M., Resnick, L., Goldman, P., Sherer, J.: Social factors that contribute to attrition in MOOCs. Proc. first ACM Conf. Learn. @ scale Conf. 197-198 (2014)

8. Gibbs, G.: Teaching more students: discussion with more students. Headington, Oxford (1992)

9. Herreid, C.F.: "Clicker" Cases: Introducing Case Study Teaching Into Large Classrooms. Journal of College Science Teaching. 36(2), 43-47 (2006)

10. Gehlen-Baum, V., Pohl, A., Weinberger, A., \& Bry, F.: Backstage-designing a backchannel for large lectures. In 21st Century Learning for 21st Century Skills. 459-464. Springer Heidelberg (2012) 